\title{
Revision und Validierung einer Skala zur Erfassung von Vertrauen in Journalismus ${ }^{1}$
}

\author{
Matthias Kohring / Jörg Matthes
}

Der Beitrag knüpft an eine in der gleichen Zeitschrift veröffentlichte Studie der Autoren zur Operationalisierung von Vertrauen in Journalismus an. Die Autoren kommen zu dem Schluss, dass die dort präsentierte Skala vor allem in Hinsicht auf konkrete Itemformulierungen revisionsbedürftig ist. Zudem wird im Gegensatz zur ersten Studie nun postuliert, dass Vertrauen in Journalismus als ein hierarchischer Faktor mit vier Faktoren erster Ordnung zu begreifen sei. An einer ersten Stichprobe $(n=487)$ wurde das revidierte Messmodell mit Hilfe einer konfirmatorischen Faktorenanalyse getestet und in einem anschließenden explorativen Zwischenschritt modifiziert. Dieses modifizierte Modell wurde an einer weiteren unabhängigen Stichprobe $(n=727)$ erneut getestet und hierbei validiert. Damit ist die Hypothese bestätigt, dass Vertrauen in Journalismus ein hierarchischer Faktor (zweiter Ordnung) ist, der die Faktoren (erster Ordnung) „Vertrauen in Themenselektivität", "Vertrauen in Faktenselektivität", "Vertrauen in die Richtigkeit von Beschreibungen" und, Vertrauen in explizite Bewertungen " erklärt. Mit der Bestätigung dieses Messmodells stebt zum ersten Mal eine validierte Skala zur Messung von Vertrauen in Journalismus zur Verfügung.

Stichwörter: Vertrauen in Journalismus, Glaubwürdigkeit, Skalenentwicklung, Validierung, konfirmatorische Faktorenanalyse

\section{Einleitung}

Dieser Beitrag knüpft an unsere Studie zur Operationalisierung von Vertrauen in Journalismus in dieser Zeitschrift an (vgl. Matthes/Kohring 2003). Diese gründete auf dem zentralen Argument, dass die kommunikations- und medienwissenschaftliche Forschung über kein theoretisch und methodisch fundiertes Messinstrument verfügt, das es ermöglicht, Vertrauen in Journalismus zu erfassen und zu analysieren. Die damals zum ersten Mal beschriebene multidimensionale Skala konnte allerdings nicht validiert werden. In dieser Forschungsnotiz wird nun über die Weiterentwicklung und Validierung der Skala zur Messung von Vertrauen in Journalismus berichtet. Zunächst werden die einzelnen Schritte der Skalenrevision beschrieben (2). Danach präsentieren wir die Ergebnisse von zwei repräsentativen Befragungen, die zur Validierung der Skala führten (3). Abschließend diskutieren wir die Verwendungsmöglichkeiten des Messinstrumentes (4).

\section{Revision der Vertrauensskala}

In unserer ersten Studie (vgl. Matthes/Kohring 2003) wurde in einer konfirmatorischen Faktorenanalyse (KFA) ein Modell für Vertrauen in Journalismus mit folgenden vier

1 Ermöglicht wurde diese umfangreiche Studie durch eine Sachbeihilfe der Deutschen Forschungsgemeinschaft (DFG-Geschäftszeichen KO 1854/2-1). 
Faktoren spezifiziert: Vertrauen in Themenselektivität, Vertrauen in Faktenselektivität, Vertrauen in die Richtigkeit von Beschreibungen und Vertrauen in explizite Bewertungen. Einer dieser Faktoren, Richtigkeit von Beschreibungen (die traditionelle ,Glaubwürdigkeit') umfasste zwei Subdimensionen bzw. Faktoren erster Ordnung: Korrektheit von Informationen und Vollständigkeit von Informationen. Nach einer Modellmodifizierung entsprachen die Fit-Indizes dem gängigen Anspruchsniveau. Da auch alternative Modellstrukturen keinen besseren Fit erzielten, konnte die postulierte Faktorstruktur als vorläufig bestätigt angesehen werden.

Die Skalenentwicklung kann damit noch nicht als abgeschlossen gelten. Die empirische Umsetzung der vier postulierten Faktoren erfolgte in der vorangegangenen Studie nicht rein konfirmatorisch: Mit Hilfe von so genannten Modifizierungsindizes wurde das Modell durch den Ausschluss von Items teilweise optimiert, d. h. besser an die empirischen Daten angepasst. Um stichprobenbedingte Fehlmodifizierungen auszuschließen, muss die extrahierte Struktur deshalb an einer unabhängigen Stichprobe erneut überprüft bzw. kreuzvalidiert werden (Kline 1998: 216 f; MacCallum/Roznowski/Necowitz 1992: 491). Sieht man von der Stichprobengröße und Stichprobenziehung in der ersten Untersuchung einmal ab (vgl. Matthes/Kohring 2003: 14), sprechen allerdings drei Gründe dagegen, das oben beschriebene Modell bereits jetzt einer Validierung zu unterziehen (vgl. auch die Empfehlungen von Reise/Waller/Comrey 2000: $288 \mathrm{ff}$ ): (1) die konkreten Itemformulierungen, (2) die rein explorativ postulierte Zuordnung des Aspektes Vollständigkeit zum Faktor Richtigkeit und (3) die bisher nicht berücksichtigte hierarchische Struktur des Vertrauens.

(1) Schon im ersten Beitrag hatten wir auf notwendige Verbesserungen bei einzelnen Itemformulierungen hingewiesen. Zunächst weisen einige Items nur verhältnismäßig geringe Ladungen auf: Andere Items waren aufgrund ihrer relativen Kompliziertheit und Länge für telefonische Umfragen ungeeignet. Möglicherweise ist auch der in der ersten Studie beobachtete Zusammenhang zwischen der Korrektheit und der Vollständigkeit von Informationen auf die spezielle Formulierung von Items zurückzuführen.

(2) Auch die explorativ gewonnene Hypothese, dass sich der hierarchische Faktor Richtigkeit von Beschreibungen aus den beiden Subdimensionen Vollständigkeit von Informationen und Korrektheit von Informationen zusammensetzt, sollte erneut überdacht werden (vgl. im Folgenden Kohring 2004: 149 ff). Die Richtigkeit von Beschreibungen bezieht sich allein auf die Überprüfbarkeit oder Konsentierbarkeit von „Fakten“. Zugunsten dieser deduktiven Ableitung der Faktorencharakteristika werden daher die Vollständigkeitsitems zum Faktor Faktenselektivität gezählt, also zu der Dimension, bei der es um die Relationierung eines Themas durch selektives Zur-Verfügung-Stellen weiterer Informationen geht.

(3) Der dritte Aspekt, der gegen einen vorschnellen Validierungsversuch des ersten Modells spricht, ist eine mögliche hierarchische Struktur des Vertrauens (vgl. Kohring 2004: 164 ff). Die hohen Korrelationen zwischen den vier Dimensionen resp. Faktoren, die sich in der ersten empirischen Überprüfung zeigten, verweisen auf eine hohe Interdependenz der Faktoren. Die Diskriminanzvalidität wurde zwar mit dem $\chi^{2}$-Differenztest überprüft (vgl. Homburg/Pflesser 2000: 429), dennoch könnte der Sinn einer inbaltlichen Unterscheidung des Vertrauens in Journalismus in gleich vier Dimensionen durchaus in Frage gestellt werden. Etwas anderes wäre es, wenn dieser hohe Zusammenhang zwischen den vier Faktoren auf einen übergeordneten Faktor Vertrauen zurückgeführt werden könnte. Dieses Vorgehen ist bei Strukturgleichungsmodellen dann üblich, wenn die entsprechenden Faktoren erster Ordnung eine hohe bis sehr hohe Korrelation aufweisen (vgl. Finch/West 1997: 456; Byrne 2001: 120 ff; Kline 1998: 233 ff). 
Ohne hierarchischen Faktor 2. Ordnung könnte der Schluss nahe liegen, dass die vier Faktoren 1. Ordnung identisch sind. Mit hierarchischem Faktor ist dagegen von der Identität ibrer Ursache auszugehen, ein entscheidender Unterschied für die Interpretation des Konstrukts. Hohe Koeffizienten deuten nun nicht mehr auf eine potenzielle Identität der einzelnen Faktoren, sondern auf die hohe Erklärungskraft des latenten übergeordneten Faktors. Die theoretische Plausibilität ist ebenfalls ein Argument, auf einen übergeordneten Faktor zweiter Ordnung zu schließen (vgl. Kohring 2001: 87; 2002: 107). Ein weiteres Argument ist pragmatischer Natur: Mit einem hierarchischen Faktor kann das Vertrauen in Journalismus mit Hilfe eines einzigen, aussagekräftigen Faktorwertes angegeben werden.

Aufgrund der vorangegangenen Überlegungen wird die bisherige Skala zunächst in einzelnen Aspekten revidiert. Die wichtigsten Änderungen sind die Einführung eines hierarchischen latenten Faktors Vertrauen in Journalismus sowie die Eingliederung der Vollständigkeitsitems unter den Faktor Vertrauen in Faktenselektivität. Die zu testende Hypothese lautet daher wie folgt:

Vertrauen in Journalismus ist ein hierarchischer Faktor (zweiter Ordnung), der die

Faktoren (erster Ordnung) „Vertrauen in Themenselektivität“, „Vertrauen in

Faktenselektivität“, „Vertrauen in die Richtigkeit von Beschreibungen “ und „Vertrauen in explizite Bewertungen " erklärt.

Zusätzlich wurden noch einmal dreißig Leitfadeninterviews geführt (vgl. Motikat 2003, Kohring 2004: 183 ff), um u. a. konkrete Itemformulierungen zu verbessern und neue Items zu generieren. Der überarbeitete standardisierte Fragebogen wurde vom Zentrum für Umfragen, Methoden und Analysen (ZUMA) zunächst einem kognitiven und danach einem telefonischen Pretest unterzogen. Von den ursprünglich 17 Items der vorangegangenen Studie wurden schließlich neun Items direkt übernommen bzw. nur leicht modifiziert. Acht Items wurden vollständig umformuliert (vgl. hierzu Clark/Watson 1995: 312 f; Osterlind 1998: 107 ff). Zunächst sollte vermieden werden, dass die Faktoren aufgrund von identischen Wörtern zustande kommen. Auch wurden diejenigen Items neu- oder umformuliert, die in der Vorgängerstudie eine geringe Ladung aufgewiesen hatten. Schließlich mussten die Items der Subdimension Vertrauen in die Korrektheit von Informationen geändert werden, da eine zu starke semantische Ähnlichkeit zu der Subdimension Vertrauen in die Vollständigkeit von Informationen festzustellen war. Vier weitere Items wurden komplett neu formuliert.

Die postulierte Faktorstruktur wurde in zwei Ende Juni 2002 durchgeführten repräsentativen telefonischen Befragungen überprüft: Die erste Befragung (Schwerin) diente als Test des neu spezifizierten Modells von Vertrauen in Journalismus. Zu erwarten ist allerdings, dass Modifikationen an dem Modell nötig sein werden. Die dergestalt modifizierte Skala soll daher an einer zweiten unabhängigen Stichprobe (Berlin) überprüft werden. Die Stichprobenziehung erfolgte mit Hilfe des Verfahrens von Gabler und Häder (vgl. 1997; 1999), das eine reine einfache Zufallsauswahl von Haushalten mit Telefonanschlüssen unter Berücksichtigung der Nichteingetragenen gestattet. Alle Befragungen wurden vorgeblich zum Thema Arbeitslosigkeit durchgeführt, zum einen, um keine Reaktivität hinsichtlich des Vertrauensthemas zu erzeugen, zum anderen, um bei den Befragten eine kognitive Referenz für ihre Antworten zur Berichterstattung zu garantieren. Die Befragten sollten jeweils im Hinblick auf das von ihnen am meisten genutzte journalistische Informationsangebot antworten. Wir waren an der Struktur von Vertrauensurteilen interessiert - diese hofften wir, vor allem bei solchen eher ausgeprägten und nicht-flüchtigen Vertrauensbeziehungen erfassen zu können.

Bei der konfirmatorischen Faktorenanalyse wird als Verfahren zur Parameterschät- 
zung (vgl. Kline 1998: 125 ff) wie auch zur Schätzung von Missings (vgl. Arbuckle/Wothke 1999: 331 ff; Kline 1998: 302 f) die (Full Information) Maximum-Likelihood-Methode verwendet. Die Daten wurden auf univariate Normalverteilung überprüft, wobei keine Abweichungen festzustellen waren (vgl. Kline 1998: 82). Zur Beurteilung der Modellgüte bzw. des Modell-Fits werden folgende gängige Maßzahlen herangezogen (vgl. Byrne 2001: 83 f; Browne/Cudeck 1993: 144; Homburg/Pflesser 2000: 430, Tab. 2; Kline 1998: 128; MacCallum/Browne/Sugawara 1996: 134): der Chi-Quadrat-Wert geteilt durch die Anzahl der Freiheitsgrade (Anspruchsniveau: $\chi 2 / d f<3$ ), der Comparative Fit Index (Anspruchsniveau: CFI $\geq .95$ ), der Root Mean Square Error of Approximation (Anspruchsniveau: RMSEA $\leq .05$ ), der PCLOSE (Mindestanspruchsniveau: PCLOSE $>$.05). Des Weiteren sollte die Indikator-Reliabilität, d. h. die durch „seinen“ Faktor erklärte Varianz eines Indikators $\geq .4$ sein.

\section{Ergebnisse}

\subsection{Modellentwicklung}

Bei der ersten Befragung in Schwerin wurden insgesamt 510 Interviews realisiert, von denen 487 gültig waren. ${ }^{2}$ Während die Gütemaße für die Beurteilung des vollständigen Strukturgleichungsmodells schon akzeptabel ausfallen, trifft dies auf der Indikatorebene noch nicht zu. Auf der Basis der so genannten Modifizierungsindizes wurden gemäß dem gängigen Vorgehen (vgl. Kline 1998: 216) daher im Laufe der weiteren Berechnung kontinuierlich fünf weitere der insgesamt 21 Variablen eliminiert. Das hieraus resultierende modifizierte Modell ist in Abb. 1 abgebildet, die Item-Formulierungen mit den dazugehörigen Faktorladungen und Indikator-Reliabilitäten sind aus Tab. 1 zu ersehen. Die Fit-Indizes für das modifizierte Modell genügen den Anspruchsniveaus alle in eindeutiger Form: $\chi^{2} / \mathrm{df}=2.407$; CFI $=.995$; RMSEA $=.044$; PCLOSE $=.914$. Ein Test dieses Modells gegen ein einfaktorielles Modell („nested model comparison“; vgl. Arbuckle/Wothke 1999: 342 ff), in dem ein einziger Faktor Vertrauen alle 16 Items erklärt, ergab, dass das hier präsentierte vierfaktorielle Modell eindeutig besser an die Daten angepasst ist.

\subsection{Modellvalidierung}

Das datengeleitete Vorgehen bei der Modifikation des Strukturgleichungsmodells machte eine Validierung der faktoriellen Struktur an einer unabhängigen Stichprobe notwendig. Die Stichprobeninvarianz wurde mit einer zeitgleich durchgeführten Befragung in Berlin überprüft, bei der von 812 Interviews 727 Fälle gültig waren. ${ }^{3}$ Die für das

2 Grund für den Ausschluss waren extrem kurze Interviewdauer bzw. extrem monotone Antwortmuster. 51,3\% der Befragten des bereinigten Samples sind weiblich, das Durchschnittsalter betrug 48,8 Jahre. Von den Befragten haben 27,3\% Abitur, 26,9\% einen Realschulabschluss, 15,4 \% einen Hauptschul- oder Volksschulabschluss und weitere 14,4 \% einen Abschluss der Polytechnischen Oberschule. 36,6 \% der Befragten haben eine Lehre absolviert, der Anteil der Universitäts- und Hochschulabsolventen beträgt 21,8 \%.

3 54,2 \% der Befragten des bereinigten Samples waren weiblich, das Durchschnittsalter betrug 46,9 Jahre. Von den Befragten haben 40,2 \% Abitur, 27,8 \% einen Realschulabschluss, 14,7 \% einen Hauptschul- oder Volksschulabschluss. 27,8 \% der Befragten haben eine Lehre absolviert, der Anteil der Universitäts- und Hochschulabsolventen liegt bei 23,8 \%. Damit sind, wie in Schwerin, höhere Ausbildungen überrepräsentiert. 

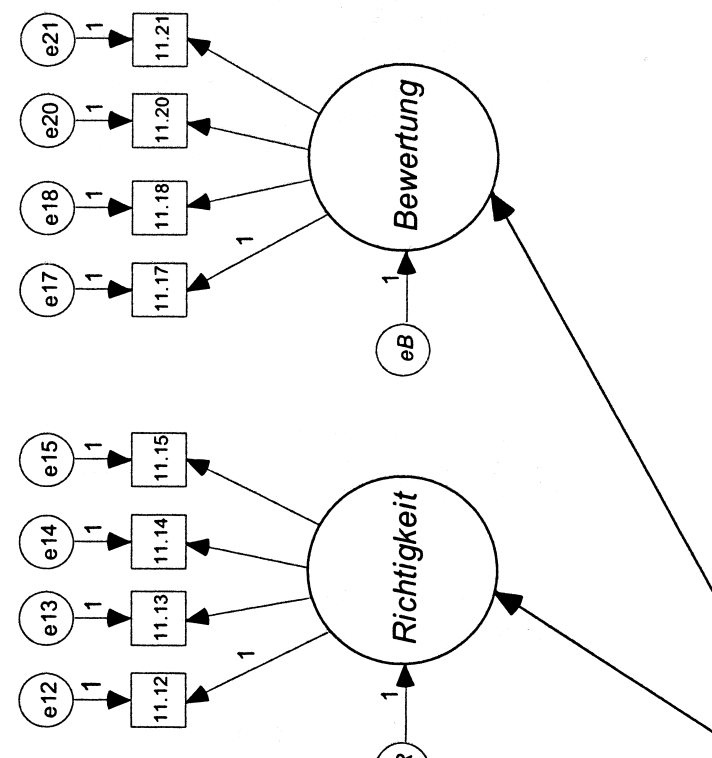

(

(a) $\rightarrow$

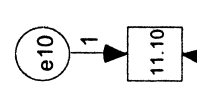

(3) $=$

(3)

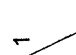

造

(4)

(8) $-\stackrel{\square}{=}$

(ङ) $\Rightarrow \stackrel{\square}{=}$

(8) $-\stackrel{m}{=}$

(c) $\Rightarrow$

$\ddot{-}$

$\stackrel{8}{\square}$

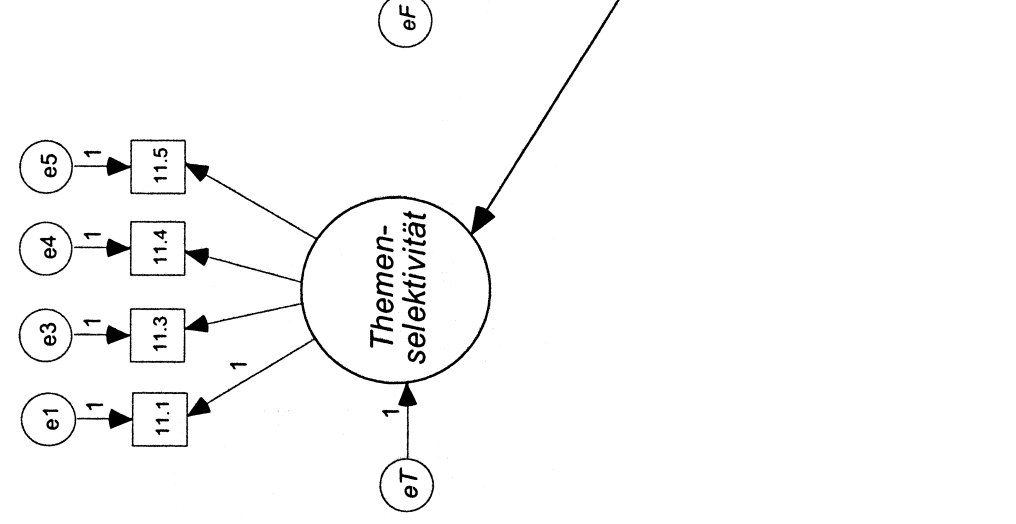


Tabelle 1: Faktorladungen (FL) und Indikator-Reliabilität (IR) der modifizierten (Schwerin) und konfirmierten Skala (Berlin)

\begin{tabular}{|c|c|c|c|c|c|}
\hline & Items & Sch & & & \\
\hline & & FL & IR & FL & IR \\
\hline & latenter Faktor & .725 & .526 & .792 & .627 \\
\hline : & $\begin{array}{l}\text { Dem Thema Arbeitslosigkeit wird die } \\
\text { nötige Aufmerksamkeit geschenkt. (11.1) }\end{array}$ & .789 & .623 & .732 & .536 \\
\hline 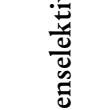 & $\begin{array}{l}\text { Dem Thema Arbeitslosigkeit wird der } \\
\text { angemessene Stellenwert zugewiesen. } \\
(11.5)\end{array}$ & .748 & .560 & .769 & .591 \\
\hline$\stackrel{\Xi}{\Xi}$ & $\begin{array}{l}\text { Die Häufigkeit, mit der über Arbeitslosig- } \\
\text { keit berichtet wird, ist angemessen. (11.3) }\end{array}$ & .689 & .475 & .709 & .503 \\
\hline & $\begin{array}{l}\text { Über das Thema wird mit der erforderlichen } \\
\text { Regelmäßigkeit informiert. (11.4) }\end{array}$ & .672 & .452 & .748 & .559 \\
\hline & latenter Faktor & .981 & .962 & .995 & .990 \\
\hline : & $\begin{array}{l}\text { Die wesentlichen Punkte werden } \\
\text { berücksichtigt. (11.9) }\end{array}$ & .710 & .504 & .709 & .503 \\
\hline 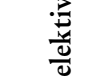 & $\begin{array}{l}\text { Wichtige Dinge stehen im Vordergrund. } \\
(11.11)\end{array}$ & .685 & .470 & .696 & .484 \\
\hline 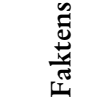 & $\begin{array}{l}\text { Zum Thema Arbeitslosigkeit werden mir } \\
\text { alle wichtigen Informationen geboten. } \\
\text { (11.10) }\end{array}$ & .658 . & 432. & .646 & .418 \\
\hline & $\begin{array}{l}\text { Die Berichterstattung erfolgt aus mehreren } \\
\text { Blickwinkeln. (11.6) }\end{array}$ & .601 & .361 & .583 & .339 \\
\hline & latenter Faktor & .868 & .753 & .857 & .743 \\
\hline 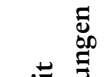 & $\begin{array}{l}\text { Die Angaben in der Berichterstattung wür- } \\
\text { den einer Überprüfung standhalten. (11.15) }\end{array}$ & .721 & .519 & .723 & .523 \\
\hline 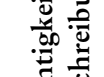 & $\begin{array}{l}\text { Die mitgeteilten Informationen sind wahr. } \\
(11.12)\end{array}$ & .715 & .512 & .742 & .550 \\
\hline 承 & $\begin{array}{l}\text { Die Berichte geben die Fakten so wieder, } \\
\text { wie sie sind. (11.13) }\end{array}$ & .706 & .499 & .757 & .573 \\
\hline & $\begin{array}{l}\text { Ich erhalte korrekte Daten über Arbeits- } \\
\text { losigkeit. (11.14) }\end{array}$ & .583 & .340 & .575 & .456 \\
\hline & latenter Faktor & .948 & .900 & .929 & .863 \\
\hline & $\begin{array}{l}\text { Wenn Kritik geäußert wird, geschieht das } \\
\text { in angemessener Weise. (11.20) }\end{array}$ & .664 & .441 & .673 & .453 \\
\hline 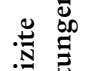 & $\begin{array}{l}\text { Die Meinungen der Journalisten sind gut } \\
\text { begründet. (11.21) }\end{array}$ & .660 & .436 & .689 & .474 \\
\hline 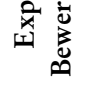 & $\begin{array}{l}\text { In den Kommentaren zur Arbeitslosigkeit } \\
\text { werden gut überlegte Schlüsse gezogen. } \\
(11.18)\end{array}$ & .657 & .432 & .694 & .482 \\
\hline & $\begin{array}{l}\text { Die journalistischen Bewertungen zum } \\
\text { Thema Arbeitslosigkeit empfinde ich als } \\
\text { nützlich. (11.17) }\end{array}$ & .625 & .390 & .595 & .355 \\
\hline
\end{tabular}


postulierte Messmodell errechneten Fit-Indices entsprechen den Anspruchsniveaus: $\chi^{2} / \mathrm{df}=1.633, \mathrm{CFI}=.997, \mathrm{RMSEA}=.036$ und $\mathrm{PCLOSE}=.998$.

Die Skala zur Messung von Vertrauen in Journalismus konnte somit an einer unabhängigen Stichprobe validiert werden. Tab. 1 listet die Faktorladungen und die erklärte Varianz (Indikatorreliabilität) der Faktoren erster Ordnung sowie der manifesten Vertrauensitems für beide Samples auf. Insgesamt deuten die verhältnismäßig hohen Faktorladungen und Indikator-Reliabilitäten (bzw. quadrierten multiplen Korrelationen) nahezu aller Items auf eine reliable Messung des Konstruktes hin. ${ }^{4}$

Der Faktor Vertranen in Themenselektivität beschreibt das Vertrauen in die Thematisierungsfunktion des Journalismus. Er umfasst vier Variablen, die sich auf die Frequenz und Kontinuität der Thematisierung von Arbeitslosigkeit sowie auf die angemessene Gewichtung des Themas im Vergleich zu anderen beziehen. Der Faktor Vertrauen in Faktenselektivität bezieht sich auf die Kontextualisierung von Ereignissen. Er umfasst vier Variablen, die sich auf die Vielfalt, Vollständigkeit und Gewichtung der Informationen beziehen, die zu einem bereits gewählten Thema zur Verfügung gestellt werden. Der Faktor Vertrauen in die Richtigkeit von Beschreibungen meint mit seinen vier Variablen ausschließlich den Aspekt der empirischen Überprüfbarkeit von Faktenangaben. Der vierte Faktor Vertrauen in explizite Bewertungen umfasst vier Variablen, die sich auf die Nachvollziehbarkeit, Nützlichkeit und Adäquatheit von journalistischen Kommentaren und Bewertungen beziehen. Vor allem Vertrauen in Faktenselektivität und Vertrauen in explizite Bewertungen werden sehr stark durch den hierarchischen Faktor Vertrauen in Journalismus erklärt und machen damit sozusagen den Kern des Vertrauens in Journalismus aus.

\section{Diskussion}

In der vorliegenden Studie wurden die Revision und Validierung einer Skala zur Erfassung von Vertrauen in Journalismus vorgestellt. $\mathrm{Zu}$ fragen ist, wie sich die Skala unter anderen Bedingungen „verhält“. Damit ist zum Ersten das Mediennutzungsprofil der Befragten und zum Zweiten das der Befragung zugrunde liegende Thema gemeint. Die Befragten sollten sich in dieser Studie jeweils nur zu dem von ihnen meist genutzten journalistischen Informationsangebot äußern. Dies lässt ein vergleichsweise sehr routinisiertes Vertrauen und somit hohe Faktorkorrelationen erwarten. Eine weitere Besonderheit war die hohe Eigenerfahrung der Befragten mit dem Thema Arbeitslosigkeit Vertrauen spielt aber eine umso größere Rolle, je mehr ein Thema sich der eigenen Erfahrung entzieht. Ein anderes Thema, z. B. aus der Auslandsberichterstattung, ließe daher ebenfalls eine stärker differenzierte Faktorenstruktur erwarten. Eine kürzlich durchgeführte weitere Überprüfung der Skala $(n=980)$, in der aktuelle und ehemalige Leser einer Regionalzeitung zur politischen Berichterstattung befragt wurden, ergab nicht nur erneut eine weitere Bestätigung der Skala, die sich damit als themen- und medienübergreifend anwendbar erweist, sondern in der Tat auch geringere Faktorkorrelationen.

4 Auch der mitunter kritisierte (vgl. Clark/Watson 1995: 316), aber dennoch gängige Koeffizient Cronbachs Alpha ist für die einzelnen Faktoren zufriedenstellend: Themenselektivität: .825 (Schwerin) bzw. .830 (Berlin); Faktenselektivität: .754 (Schwerin) bzw. 758 (Berlin); Richtigkeit von Beschreibungen: 769 (Schwerin) bzw. .817 (Berlin); Explizite Bewertungen: .742 (Schwerin) bzw. .754 (Berlin). 
Das hier vorgestellte Erhebungsinstrument ist damit die erste theoretisch abgeleitete, sukzessiv entwickelte sowie an unabhängigen Stichproben validierte Skala in der kommunikationswissenschaftlichen Vertrauensforschung resp. Glaubwürdigkeitsforschung. Die bisherige Medienglaubwürdigkeitsforschung ist bei der Skalenkonstruktion weitgehend induktiv und ohne methodische Überlegungen zur Operationalisierung von komplexen Konstrukten vorgegangen (vgl. Wirth 1999: 51). Um ein einheitliches Konzept von Vertrauen in Journalismus zu etablieren und empirisch zu belegen, bedarf es aber keiner wiederholten explorativen - wie immer noch in vielen kommunikationswissenschaftlichen Skalenentwicklungen üblich (vgl. hierzu Holbert/Stephenson 2002) -, sondern letztlich einer konfirmatorischen Herangehensweise, wie hier geschehen. Die vorgestellte Skala ermöglicht nicht nur eine reliable Messung von Vertrauen, sondern auch eine umfassende und valide Beschreibung von Vertrauensurteilen. Damit ergeben sich vielfältige Einsatzmöglichkeiten der Skala, so beispielsweise der Vergleich von Vertrauenswerten verschiedener journalistischer Organe, die gezielte Diagnose und Therapie von Vertrauensdefiziten, die längerfristige Beobachtung des „Medienvertrauens“ zur Diagnose gesellschaftlicher Prozesse und nicht zuletzt die noch ausstehende funktionale Einbindung des Konstruktes in die Medienwirkungsforschung.

\section{Literatur}

Arbuckle, James L./Wothke, Werner (1999): Amos 4.0 user's guide. Chicago (IL).

Browne, Michael W./Cudeck, Robert (1993): Alternative ways of assessing model fit. In: Bollen, Kenneth A./Long, J. Scott (Hrsg.): Testing structural equation models. Newbury Park/London/New Delhi, S. 136-162.

Byrne, Barbara M. (2001): Structural equation modeling with AMOS. Basic concepts, applications, and programming. Mahwah (NJ)/London.

Clark, Lee Anna/Watson, David (1995): Constructing validity: basic issues in objective scale development. In: Psychological Assessment, 7. Jg., S. 309-319.

Finch, John F./West, Stephen G. (1997): The investigation of personality structure: statistical models. In: Journal of Research in Personality, 31. Jg., S. 439-485.

Gabler, Siegfried/Häder, Sabine (1997): Überlegungen zu einem Stichprobendesign für Deutschland. In: ZUMA-Nachrichten, 21. Jg., Nr. 41, S. 7-18.

Gabler, Siegfried/Häder, Sabine (1999): Erfahrungen beim Aufbau eines Auswahlrahmens für Telefonstichproben in Deutschland. In: ZUMA-Nachrichten, 23. Jg., Nr. 44, S. 45-61.

Holbert, R. Lance/Stephenson, Michael T. (2002): Structural equation modeling in the communications sciences. In: Human Communication Research, 28. Jg., S. 531-551.

Homburg, Christian/Pflesser, Christian (2000): Konfirmatorische Faktorenanalyse. In: Herrmann, Andreas/Homburg, Christian (Hrsg.): Marktforschung. Methoden, Anwendungen, Praxisbeispiele. 2., aktualisierte Auflage. Wiesbaden, S. 413-437.

Kline, Rex B. (1998): Principles and practice of structural equation modeling. London.

Kohring, Matthias (2001): Vertrauen in Medien - Vertrauen in Technologie. Stuttgart.

Kohring, Matthias (2002): Vertrauen in Journalismus. In: Scholl, Armin (Hrsg.): Systemtheorie und Konstruktivismus in der Kommunikationswissenschaft: Ansprüche und Herausforderungen. Konstanz, S. 91-110.

Kohring, Matthias (2004): Vertrauen in Journalismus. Theorie und Empirie. Konstanz.

Matthes, Jörg/Kohring, Matthias (2003): Operationalisierung von Vertrauen in Journalismus. In: Medien \& Kommunikationswissenschaft, 51. Jg., S. 5-23.

MacCallum, Robert C./Roznowski, Mary/Necowitz, Lawrence B. (1992): Model modification in covariance structure analysis: the problem of capitalization on chance. In: Psychological Bulletin, 111. Jg., S. 490-504. 
MacCallum, Robert C./Browne, Michael W./Sugawara, Hazuki M. (1996): Power analysis and determination of sample size for covariance structure modeling. In: Psychological Methods, 1. Jg., S. 130-149.

Motikat, Franziska (2003): Gründe für Vertrauen in Medien. Unveröffentlichte Magistra-Arbeit. Friedrich-Schiller-Universität Jena, Fakultät für Sozial- und Verhaltenswissenschaften, Bereich Medienwissenschaft.

Osterlind, Steven J. (1998): Constructing test items: multiple-choice, constructed-response, performance, and other formats. Boston.

Reise, Steven P./Waller, Niels G./Comrey, Andrew L. (2000): Factor analysis and scale revision. In: Psychological Assessment, 12. Jg., S. 287-297.

Wirth, Werner (1999): Methodologische und konzeptionelle Aspekte der Glaubwürdigkeitsforschung. In: Rössler, Patrick/Wirth, Werner (Hrsg.): Glaubwürdigkeit im Internet. Fragestellungen, Modelle, empirische Befunde. München, S. 47-66. 